Int. J. Dev. Biol. 52: 81-85 (2008)

doi: $10.1387 / \mathrm{ijdb} .072355$ th

\title{
Expression of Shisa2, a modulator of both Wnt and Fgf signaling, in the chick embryo
}

\author{
THOMAS A. HEDGE and IVOR MASON* \\ MRC Centre for Developmental Neurobiology, King's College London, Guy's Campus, London, U.K.
}

\begin{abstract}
Shisa proteins are a recently-identified family of modulators of both FGF and Wnt signaling that block both maturation and transport to the cell surface of their respective receptors. The latter are retained within the endoplasmic reticulum, thereby inhibiting or reducing cellular responses to the ligands. We describe expression of a Shisa2 orthologue in an amniote: the chick embryo. We show that Shisa2 transcripts are expressed in a dynamic manner along the anteroposterior axis, in a manner consistent with a role in head development as demonstrated for Xenopus Shisa, being ubiquitously expressed in anterior tissues. However, expression is progressively restricted anteriorly within the developing neural tube and adjacent mesenchyme and ectoderm, eventually becoming restricted to the telencephalic lobes. Similarly, from being ubiquitous within the optic cups, transcripts become restricted to the prospective ciliary margin. A similar process is evident in the somites, where expression is initially ubiquitous but remains at high levels first in dermamyotome and subsequently is only detected in myotome. During the initial stages of organogenesis, Shisa2 transcripts are detected in cardiac and lung bud mesenchyme and in nephric ducts and tubules. Within the pharyngeal region, expression is observed in pharyngeal pouches from their first appearance and later in mesenchyme of all pharyngeal arches, as well as in cranial ganglia. Transcripts are also detected in the dorsal mesenchyme of the limb bud.
\end{abstract}

KEY WORDS: endoplasmic reticulum, posteriorisation, ciliary marginal zone

Members of the Wnt and FGF families of secreted signaling proteins regulate multiple developmental processes, frequently being deployed together to instruct morphogenesis (Logan and Nusse, 2004, Thisse and Thisse, 2005). A feature of Wnt, FGF and other signaling pathways is the utilisation of extracellular and intracellular modulators, which serve to control the duration, strength and spatial extent of their action. Most commonly these are antagonists, often part of negative feedback loops, although some serve to amplify the signals (Dailey et al., 2005, Kawano and Kypta, 2003, Logan and Nusse, 2004, Tsang and Dawid, 2004). However, a novel regulatory mechanism, mediated by an endoplasmic reticulum-resident protein, Shisa, has recently been identified in Xenopus (Yamamoto et al., 2005). Shisa regulates the post-translational modification and trafficking to the cell surface of receptors for both Wnts (frizzled proteins; Fz) and FGFs (FGFRs). Inhibition studies in that organism revealed an early essential role for Shisa in head development through its suppression of responses to these caudalising factors (Yamamoto et al.,
2005). A recent study has shown that all vertebrates have at least four Shisa (Shisa2-5) genes in their genomes, with zebrafish and Xenopushaving an additional member; the prototypic Shisa (now Shisa1) (Furushima et al., 2007). The biochemical studies described above were performed using the latter, however murine Shisa2 has been shown to regulate Wnt and FGF activity in a similar manner to Xenopus Shisa1 (Furushima et al., 2007).

Our interrogation of the first draft of the chicken genome identified a single Shisa orthologue on chromosome 6 within contig 9.365. Sequences from that region were used to identify a chicken cDNA in the Genbank database (accession number NM 204501), a corresponding EST was obtained and identified as the chicken Shisa2 orthologue (Furushima et al., 2007). This EST was used to characterize Shisa2 expression between gastrulation and limb bud stages of development (Fig. 1 A-R, Fig. 2 A-P).

At the earliest stage examined, stage 4 of Hamburger and Hamilton (1951; HH4), Shisa2 mRNA was detected in the anterior

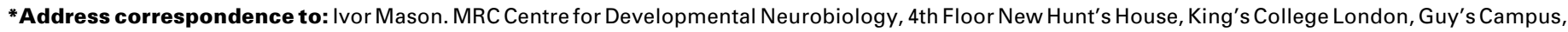
London, SE1 1UL, U.K. Tel: +44-(0)-207-848-8148. Fax: +44-(0)-207-848-6798. e-mail: ivor.mason@kcl.ac.uk

Website: http://www.kcl.ac.uk/depsta/biomedical/mrc/ResearchGroup.php?GroupID=20
}

Accepted: 18th July 2007. Published online: 13th November 2007.

$0214-6282 / 2008 / \$ 35.00$

(C) UBC Press

Printed in Spain 
Fig. 1. Shisa2 expression between gastrulation and neural segmentation stages. In situ hybridisation to embryos at (A) $\mathrm{HH}_{4}$, (B) HH5, (C-E) HH6, (F-J) HH8, (K) HH1O and (L-R) HH12. (A-C,F,K-M) Whole embryo preparations. (D,E) Transverse sections of an HH6 embryo taken at the levels indicated in (C). (G-J) Transverse sections of an HH8 embryo taken at the levels indicated in (F). Reduced abundance of Shisa2 transcripts in dorsal neural folds and ventral endoderm and ectoderm are indicated in $\mathbf{( H )}$ by an arrowhead and arrow respectively. Arrow in (K) indicates cardiac mesenchyme in an embryo viewed ventrally. (L) Inset: higher magnification of trunk region to show expression in intermediate mesoderm adjacent to somites. Arrowhead indicates transcripts in the pharyngeal region, arrow indicates expression in cardiac mesenchyme. (M) Higher magnification image of the anterior region of the embryo in (L); arrow indicates prechordal mesoderm and arrowhead indicates the head process. (N) Longitudinal section through an optic lobe; reduced mRNA levels in the posterior diencephalon are indicated larrow; see also Fig. 2B) while transcripts are undetectable in mesenchyme posterior to the optic lobe. (O-R) Transverse sections of an HH12 embryo taken at the levels indicated in (L) Arrowhead indicates ectoderm and arrow indicates mesoderm associated with pharyngeal pouches in (O).
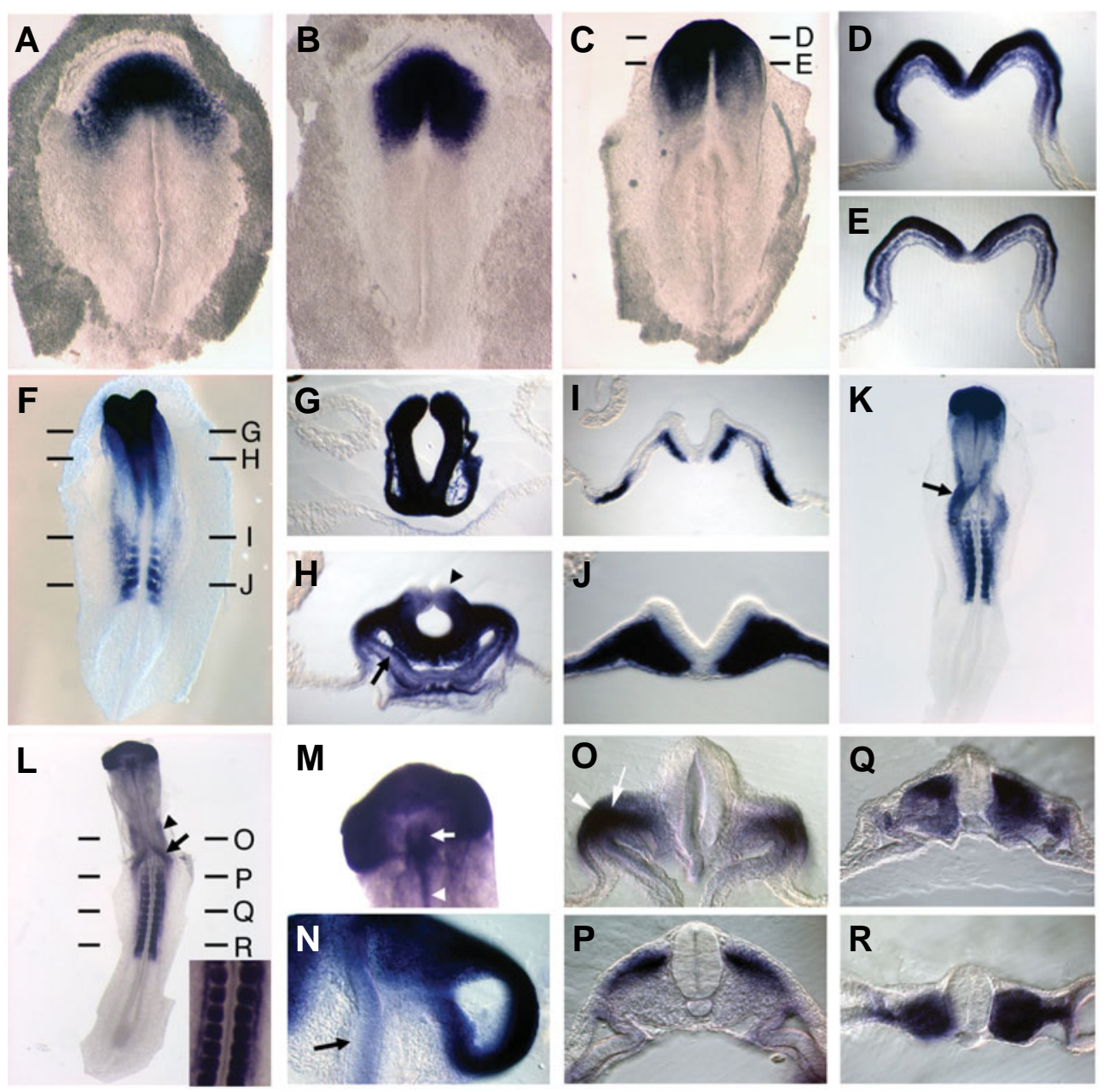

of the embryo (Fig. 1A) and this pattern of expression persisted during early stages of node regression (Fig. 1 B,C). Sections of embryos at these stages showed the presence of transcripts in all three germ layers, with expression being particularly prominent in the ectoderm and neural plate (Fig. 1 D,E). Comparison with fate maps of the neural plate at HH4 (Cobos et al., 2001) indicated that transcripts were present in prospective forebrain, midbrain and hindbrain and, overall ectodermal expression was most reminiscent of Otx2 at that stage (Bally-Cuif et al., 1995, Puelles et al., 2005) and complementary to that of Gbx2 (Shamim and Mason, 1998). Thereafter, expression in the neural plate and neural tube was dynamic and characterized by a progressive rostral shift in the caudal limit of Shisa2 expression. A recent study of mouse Shisa2 expression also reported that it was co-extensive with Otx2 at neural plate stages. Moreover ectopic expression in Xenopus resulted in expansion of the Otx2 domain and Shisa2 transcripts were reduced or absent in Otx2null mice (Furushima et al., 2007).

During early neurulation stages mRNA was detected in a domain that is fated to give rise to forebrain and midbrain, consistent with co-expression with Otx2 (Fig. 1F; (Couly and Le Douarin, 1985); in anterior regions transcripts were present throughout the neuroepithlium and in all other tissues at uniformly high levels (Fig. 1G). However, more posteriorly, reduced levels of expression were observed in the dorsal neural tube and in ventral ectoderm and endoderm lining the floor of the pharynx (Fig. 1H).

As the neuromeric architecture of the developing brain became apparent at HH10 (10 somites), Shisa transcripts were further restricted to the prospective diencephalon and telencephalon and their adjacent ectoderm. Transcripts were also detected in anterior head process and prechordal mesoderm and, at lower levels, in mesenchyme adjacent to the midbrain (Fig. $1 \mathrm{~K}$ and data not shown). Approximately $12 \mathrm{hr}$ later $(\mathrm{HH} 12)$, expression remained elevated in the optic lobes and prospective telencephalon while becoming reduced in the posterior diencephalon, although abundant transcripts were still detected in underlying prechordal mesoderm and anterior head process (Fig. $1 \mathrm{~L}-\mathrm{N}$ ). Transcripts were now undetectable in head mesenchyme posterior to the optic cups (Fig. 1N). Shortly thereafter Shisa2RNA became restricted within the brain to the telencephalon and optic lobes (Fig. 2 A,B). As development proceeded, there was further apparent reduction in transcript levels within the telencephalon (Fig. 2 D,E,L), such that by $\mathrm{HH} 21$ highest RNA levels were detected in the lateral telencephalic vesicles (Fig. 2 L,M).

Within the developing ocular system, abundant transcripts were detected in the developing retina and lens but became reduced in the optic stalks (HH15; Fig. $2 \mathrm{D}, \mathrm{F})$. However, at later stages $(H H 19,21)$ apparent expression was confined to the prospective ciliary marginal zone, a source of retinal stem cells in 
the chick (Kubota et al., 2004, Moshiri et al., 2004) and was undetectable throughout the retina or within the lens (Fig. 2 E,G,L,O). Notably, both FGF and Wnt signaling has been implicated in regulation of retinal development and regeneration at the ciliary margin (Liu et al., 2007, Spence et al., 2004). In the oldest embryos examined, apparent, weak expression was detected in Rathke's pouch (data not shown) and in cranial ganglia (Fig. 2L; data not shown) but not in other components of the peripheral nervous system.

Somites are subject to instructive signaling by both FGFs and Wnts (see e.g. Brent and Tabin, 2004, Linker et al., 2005 and references therein) and Shisa2 transcripts were detected in developing somites from the onset of somitogenesis (Fig. $1 \mathrm{~F}, \mathrm{~K}$, L, Fig. 2 A,D,E, L) and, as in the brain, exhibited a dynamic pattern of expression during their maturation. Initially, RNA was detected throughout recently-cleaved, epithelial somites (Fig. $1 \mathrm{~J}, \mathrm{R}$ ). As maturation proceeded, transcripts became progressively restricted, first to the dermamyotome (Fig. 1 P,Q, Fig. 2 C,P) and eventually to the myotome only (Fig. 2 I and data not shown). At early stages, expression was also found in lateral plate mesoderm (Fig. $1 \mathrm{I}, \mathrm{J}$ ) but this did not persist (see e.g. Fig. 1P). Within the head at $\mathrm{HH} 19$, transcripts were detected medial to the trigeminal ganglion in cells identified by others as the precursors of the lateral rectus muscle of the eye at that stage of development (Fig. 2 E,H) (Mootoosamy and Dietrich, 2002).

Within the posterior of the embryo Shisa2 mRNA was undetectable within the primitive streak, tail bud and segmental plate at all stages (Fig. 1 A-C,F,K,L; Fig. 2 A,D,E,L) and, while expression was detected anteriorly within the head process and prechordal mesoderm (see above), transcripts were never detected in the notochord (Fig. $1 \mathrm{~J}, \mathrm{P}-\mathrm{R}$, Fig. $2 \mathrm{H}, \mathrm{I}, \mathrm{K}, \mathrm{P}$ ). Shisa2 was observed in cardiac mesenchyme at early somitogenesis stages (Fig. $1 \mathrm{~K}, \mathrm{~L}$ ) and later in mesenchyme in the region of the developing lung buds (Fig. 2 A,D,E,J,L). Transcripts were also detected in intermediate mesoderm (Fig. $1 \mathrm{~L}, \mathrm{Q}$ ) and subsequently in nephric ducts (Fig. 2C) and mesonephric tubules (Fig. 2N). At early stages of limb bud outgrowth, expression was undetectable in the buds (Fig. 2K), however at the oldest stages analysed, RNA was detected in the dorsal mesenchyme (Fig. 2P) a region known to be subject to Wnt signaling (Kengaku et al., 1998).
Fig. 2. Shisa2 expression between flexure and limb bud stages. In situ hybridisation to embryos at (A-C) HH13, (D,F) HH15, (E, G-K) HH19 and (L-P) HH21. (A,D,E,L) Whole embryo preparations. (B) Coronal section through the forebrain, anterior to the top; note lack of detectable transcripts in the diencephalic tissue (arrow). (C) Transverse section through an anterior somite showing elevated expression in dermamyotome (arrow) compared to adjacent sclerotome. Arrowhead indicates transcripts associated with the nephric duct. $(\mathbf{F}, \mathbf{G})$ Coronal section taken through the retina and lens at HH15 (F) and HH19 (G). (H) Transverse section showing expression in cells at the position of precursors to the lateral rectus muscle of the eye. (I) Transverse section taken at the level of the posterior hindbrain showing expression restricted to the myotome. (J) Mesenchymal expression in the region of the developing lung buds. (K) Transverse section at the level of the wing bud. (M) Coronal section taken through the telencephalon. (N) Longitudinal section showing expression in tubules of the mesonephros. (0) Longitudinal section taken through the pharyngeal region. Arrowhead indicates expression in the prospective ciliary body and arrow indicates expression in the third pharyngeal pouch. Abbreviations: Di, diencephalon; $M d$, mandibular process; Mx, Maxilliary process; II,III,IV, second, third and fourth pharyngealarches respectively. (P) Transverse section taken at the level of the hindlimb bud. Arrowhead indicates dermamyotome and arrow indicates dorsal limb bud mesenchyme.
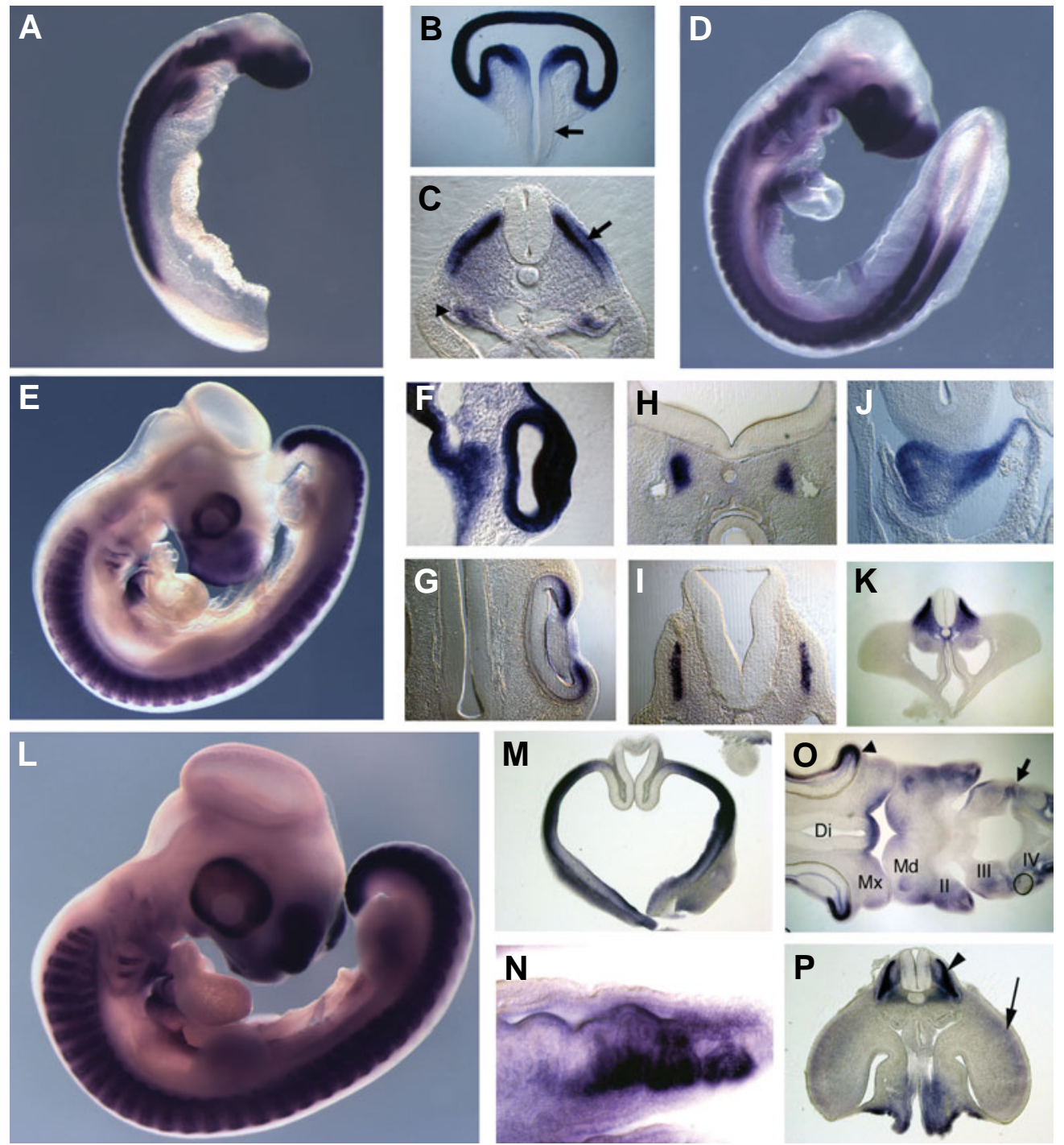
Transcripts were detected within the pharyngeal region adjacent to the developing hindbrain ( $\mathrm{HH} 12$; Fig. $1 \mathrm{~L})$, a prominent site of Fgf expression in the avian embryo (see e.g. Mahmood et al., 1995; Shamim and Mason 1999; Shamim et al., 1999a) and were particularly abundant in the mesenchyme and ectoderm adjacent to the pharyngeal pouches (Fig. 1O). Expression persisted in the pouches at later stages (Fig. 2 E,L,O) and was also detected in mesenchyme of the maxilliary and mandibular lobes of the first pharyngeal arch and in all posterior arches (Fig. 2O).

In summary, Shisa2 transcripts were detected in many tissues that are known to be influenced by FGF and Wnt signaling. Our findings are similar but not identical to those reported by others for avian Shisa2, although that study was not as detailed as this one, particularly at later stages (Filipe et al., 2006). A brief report of expression of the mouse Shisa2 orthologue at similar stages to those used in our study indicates that its pattern of expression is conserved among amniotes (Furushima et al., 2007). By contrast, expression of the Xenopus Shisa 1orthologue seems more similar to amniote Shisa2 than the Xenopus Shisa2 cognate (Silva etal., 2006).

It is noteworthy that our study reveals that the relationship between Shisa2 expression and Wnt- or FGF-dependent processes is not absolute, for example it does not appear to be associated with gastrulation movements, initial stages of limb bud outgrowth, otic induction or patterning signals from the midhindbrain boundary; processes regulated by both types of ligand. Furthermore, it cannot be considered to be part of a synexpression group for either family, unlike many other antagonists. The studies of murine Shisa3-5 published to date indicate that this is not due to differential sub-functionalisation during evolution (Furushima et al., 2007).

Axial expression from earliest stages is consistent with the proposed role for the Shisas in antagonizing posteriorizing reponses to FGF and Wnt signals in Xenopus (Yamamoto et al., 2005) and progressive restriction to anterior regions indicates that competence to respond to them is temporally modulated. Likewise, dynamic spatial and temporal expression during somitic maturation and differentiation is indicative of altering responses to extracellular cues during their development. However, mice homozygous for a targeted null mutation in Shisa2 have normal early development, although the majority are reduced in size at birth. The authors argue against compensation by other Shisa genes, although other mechanisms are possible (Furushima et al., 2007).

\section{Experimental Procedures}

A chicken Shisa2orthologue was identified by interrogation of the first draft release of the chicken genome (http:// www.ensembl.org/Gallus_gallus/index.html) using the TBLASTN algorithm. The sequences recognized were used to identify a chicken EST (ChEST99402) in the BBSRC ChickEST database (http://www.chick.umist.ac.uk/), which was then obtained through Geneservice (http://www.geneservice.co.uk/). Embryos were staged according to (Hamburger and Hamilton, 1951) and in situ hybridization was performed as described (Henrique et al., 1995). Embryos were embedded in gel albumen and sectioned on a vibrotome as described (Shamim et al., 1999).

\section{Acknowledgements}

This work was supported by grants from the Medical Research Council and The Wellcome Trust. TAH is supported by an MRC studentship award and IM is a Research Fellow of the Leverhulme Trust.

\section{References}

BALLY-CUIF, L., GULISANO, M., BROCCOLI, V. and BONCINELLI, E. (1995). Cotx2 is expressed in two different phases of gastrulation and is sensitive to retinoic acid treatment in chick embryo. Mech Dev 49: 49-63.

BRENT, A.E. and TABIN, C.J. (2004). Fgf acts directly on the somitic tendon progenitors through the ets transcription factors pea3 and erm to regulate scleraxis expression. Development 131: 3885-96.

COBOS, I., SHIMAMURA, K., RUBENSTEIN, J.L., MARTINEZ, S. and PUELLES, L. (2001). Fate map of the avian anterior forebrain at the four-somite stage, based on the analysis of quail-chick chimeras. Dev Bio/239: 46-67.

COULY, G.F. and LE DOUARIN, N.M. (1985). Mapping of the early neural primordium in quail-chick chimeras. I. Developmental relationships between placodes, facial ectoderm and prosencephalon. Dev Bio/110: 422-39.

DAILEY, L., AMBROSETTI, D., MANSUKHANI, A. and BASILICO, C. (2005). Mechanisms underlying differential responses to fgf signaling. Cytokine Growth Factor Rev 16: 233-47.

FILIPE, M., GONCALVES, L., BENTO, M., SILVA, A.C. and BELO, J.A. (2006). Comparative expression of mouse and chicken shisa homologues during early development. Dev Dyn 235: 2567-73.

FURUSHIMA, K., YAMAMOTO, A., NAGANO, T., SHIBATA, M., MIYACHI, H., ABE, T., OHSHIMA, N., KIYONARI, H. and AIZAWA, S. (2007). Mouse homologues of shisa antagonistic to wnt and fgf signalings. Dev Bio/306: 480-92.

HAMBURGER, V. and HAMILTON, H. (1951). A series of normal stages in the development of the chick embryo. J. Morphol. 88: 49-92.

HENRIQUE, D., ADAM, J., MYAT, A., CHITNIS, A., LEWIS, J. and ISH-HOROWICZ, D. (1995). Expression of a delta homologue in prospective neurons in the chick. Nature 375: 787-90

KAWANO, Y. and KYPTA, R. (2003). Secreted antagonists of the wnt signalling pathway. J Cel/ Sci116: 2627-34.

KENGAKU, M., CAPDEVILA, J., RODRIGUEZ-ESTEBAN, C., DE LA PENA, J., JOHNSON, R.L., BELMONTE, J.C. and TABIN, C.J. (1998). Distinct wnt pathways regulating aer formation and dorsoventral polarity in the chick limb bud. Science 280: 1274-7.

KUBOTA, R., MCGUIRE, C., DIERKS, B. and REH, T.A. (2004). Identification of ciliary epithelial-specific genes using subtractive libraries and cDNA arrays in the avian eye. Dev Dyn 229: 529-40.

LINKER, C., LESBROS, C., GROS, J., BURRUS, L.W., RAWLS, A. and MARCELLE, C. (2005). Beta-catenin-dependent wnt signalling controls the epithelial organisation of somites through the activation of paraxis. Development 132: 3895-905.

LIU, H., XU, S., WANG, Y., MAZEROLLE, C., THURIG, S., COLES, B.L., REN, J.C. TAKETO, M.M., VAN DER KOOY, D. and WALLACE, V.A. (2007). Ciliary margin transdifferentiation from neural retina is controlled by canonical wnt signaling. Dev Biol.

MAHMOOD, R., KIEFER, P., GUTHRIE, S., DICKSON, C. and MASON, I. (1995). Multiple roles for fgf-3 during cranial neural development in the chicken. Development 121: 1399-1410.

LOGAN, C.Y. and NUSSE, R. (2004). The wnt signaling pathway in development and disease. Annu Rev Cell Dev Bio/20: 781-810.

MOOTOOSAMY, R.C. and DIETRICH, S. (2002). Distinct regulatory cascades for head and trunk myogenesis. Development 129: 573-83.

MOSHIRI, A., CLOSE, J. and REH, T.A. (2004). Retinal stem cells and regeneration. Int J Dev Bio/48: 1003-14.

PUELLES, L., FERNANDEZ-GARRE, P., SANCHEZ-ARRONES, L., GARCIA CALERO, E. and RODRIGUEZ-GALLARDO, L. (2005). Correlation of a chicken stage 4 neural plate fate map with early gene expression patterns. Brain Res Brain Res Rev 49: 167-78. 
SHAMIM, H. and MASON, I. (1999). Expression of fgf4 during early development of the chick embryo. Mech Dev 85: 189-92.

SHAMIM, H., MAHMOOD, R., LOGAN, C., DOHERTY, P., LUMSDEN, A. and MASON, I. (1999a). Sequential roles for fgf4, en1 and fgf8 in specification and regionalisation of the midbrain. Development 126: 945-59.

SHAMIM, H., MAHMOOD, R. and MASON, I. (1999b). In situ hybridization to rna in whole embryos. Methods Mol Bio/97: 623-33.

SHAMIM, H. and MASON, I. (1998). Expression of gbx-2 during early development of the chick embryo. Mech. Dev.

SILVA, A.C., FILIPE, M., VITORINO, M., STEINBEISSER, H. and BELO, J.A. (2006). Developmental expression of shisa-2 in Xenopus laevis. Int J Dev Biol 50: 575-9.
SPENCE, J.R., MADHAVAN, M., EWING, J.D., JONES, D.K., LEHMAN, B.M. and DEL RIO-TSONIS, K. (2004). The hedgehog pathway is a modulator of retina regeneration. Development 131: 4607-21.

THISSE, B. and THISSE, C. (2005). Functions and regulations of fibroblast growth factor signaling during embryonic development. Dev Bio/287: 390-402.

TSANG, M. and DAWID, I.B. (2004). Promotion and attenuation of fgf signaling through the ras-mapk pathway. SCi. STKE. 6: pe17.

YAMAMOTO, A., NAGANO, T., TAKEHARA, S., HIBI, M. and AIZAWA, S. (2005). Shisa promotes head formation through the inhibition of receptor protein maturation for the caudalizing factors, wnt and fgf. Cel/120: 223-35.

\section{Related, previously published Int. J. Dev. Biol. articles}

See our Special Issue Ear Development edited by Fernando Giraldez and Bernd Fritzsch at: http://www.ijdb.ehu.es/web/contents.php?vol=51\&issue=6-7

Wnt signaling in hydroid development: ectopic heads and giant buds induced by GSK-3beta inhibitors Werner Müller, Uri Frank, Regina Teo, Ofer Mokady, Christina Guette and Günter Plickert Int. J. Dev. Biol. (2007) 51: 211-220

Developmental expression of Shisa-2 in Xenopus laevis

Ana-Cristina Silva, Mário Filipe, Marta Vitorino, Herbert Steinbeisser and José-António Belo Int. J. Dev. Biol. (2006) 50: 575-579

The zic1 gene is an activator of Wnt signaling

Christa S. Merzdorf and Hazel L. Sive

Int. J. Dev. Biol. (2006) 50: 611-617

The Wnt connection to tumorigenesis

Jürgen Behrens and Barbara Lustig

Int. J. Dev. Biol. (2004) 48: 477-487

Flamingo, a cadherin-type receptor involved in the Drosophila planar polarity pathway, can block signaling via the canonical wnt pathway in Xenopus laevis.

Richard Morgan, Ali-Morsi El-Kadi and Christopher Theokli Int. J. Dev. Biol. (2003) 47: 245-252

Bmp, Fgf and Wnt signalling in programmed cell death and chondrogenesis during vertebrate limb development: the role of Dickkopf-1.

Lars Grotewold and Ulrich Rüther

Int. J. Dev. Biol. (2002) 46: 943-947

Interactions between dorsal-ventral patterning genes Imx1b, engrailed-1 and wnt-7a in the vertebrate limb.

Haixu Chen and Randy L Johnson

Int. J. Dev. Biol. (2002) 46: 937-941

Wnt signalling during limb development.

Vicki L Church and Philippa Francis-West

2006 ISI ${ }^{\star \star}$ Impact Factor $=3.577^{\star *}$

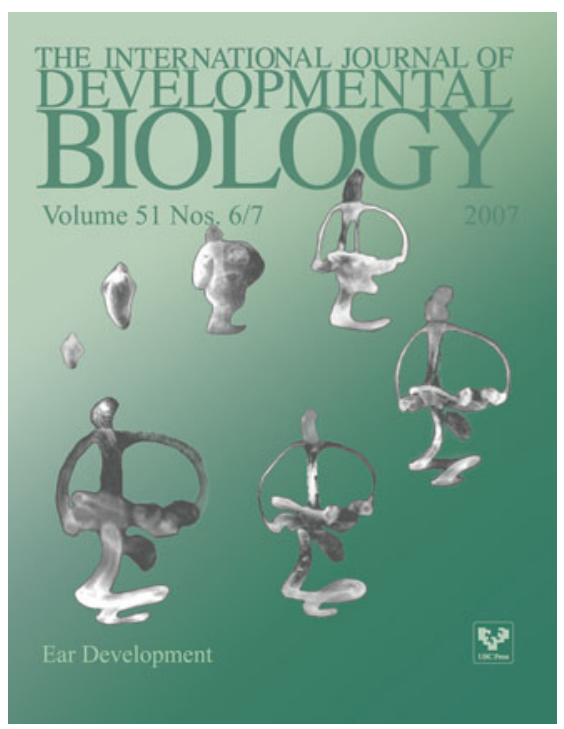

